\title{
Anesthesia-Induced Neurodegeneration in Fetal Rat Brains
}

\author{
SHOUPING WANG, KELLY PERETICH, YIFAN ZHAO, GE LIANG, QINGCHENG MENG, AND HUAFENG WEI
}

\author{
Department of Anesthesiology and Critical Care [S.W., K.P., Y.Z., G.L., Q.M., H.W.], University of Pennsylvania, Philadelphia, \\ Pennsylvania 19104; Department of Anesthesia [S.W., Y.Z.], Second Affiliated Hospital of Sun Yat-sen University, Guangzhou 510120, \\ China; Drexel University College of Medicine [K.P.], Philadelphia, Pennsylvania
}

\begin{abstract}
We investigated the extent of isoflurane-induced neurodegeneration on the fetuses of pregnant rats exposed in utero. Pregnant rats at gestational d 21 were divided into three experimental groups. Rats in the control group spontaneously breathed $100 \%$ oxygen for $1 \mathrm{~h}$. Rats in the treatment groups breathed either 1.3 or $3 \%$ isoflurane in $100 \%$ oxygen through an endotracheal tube, with mechanical ventilation for $1 \mathrm{~h}$. Rat pups were delivered by cesarian section $6 \mathrm{~h}$ after treatment, and fetal blood was sampled from the left ventricle of each fetal heart and evaluated for $\mathrm{S} 100 \beta$. Fetal brains were then evaluated for apoptosis, using caspase-3 immunohistochemistry in the CA1 region of the hippocampus and the retrosplenial cortex (RS). The 3\% isoflurane treatment group showed significantly higher levels of $\mathrm{S} 100 \beta$ levels and significantly increased average densities of total caspase-3-positive cells in the CA1 hippocampus and RS cortex compared with the control and the $1.3 \%$ isoflurane groups. There were no differences in $\mathrm{S} 100 \beta$ levels or densities of caspase-3-positive cells between the control and $1.3 \%$ isoflurane groups. Isoflurane at a concentration of $3 \%$ for $1 \mathrm{~h}$ increased neurodegeneration in the hippocampal CA1 area and the retrosplenial cortex in the developing brain of fetal rats. (Pediatr Res 66: 435-440, 2009)
\end{abstract}

$\mathrm{I}_{\mathrm{n}}^{\mathrm{n}}$ ncreasing evidence suggests that commonly used inhalational anesthetics, especially isoflurane, dose- and timedependently induce damage in various types of tissues and cells, including hippocampal slices (1), lymphocytes $(2,3)$, neuroglioma (4), liver cells (5), gingival fibroblasts (6), neurosecretory PC12 cells $(7,8)$, and primary cortical and striatal neurons $(2,7,8)$. In animal studies, isoflurane, alone or in combination with midazolam or nitrous oxide, caused widespread neurodegeneration in the 7-d-old rodent brain and was associated with subsequent cognitive impairment $(9,10)$. In the elderly rodent, isoflurane exposure also caused persistent memory impairment (11). Recent clinical studies suggest a greater incidence of learning disabilities in children exposed to anesthesia during surgery before the age of 3, especially in children over the course of multiple surgeries $(12,13)$.

Pregnant women sometimes require general anesthesia for various surgeries, such as cesarian sections and fetal surgeries.

Received March 24, 2009; accepted May 28, 2009.

Correspondence: Huafeng Wei, M.D., Ph.D., Department of Anesthesiology and Critical Care, University of Pennsylvania School of Medicine, 305 John Morgan Building, 3620 Hamilton Walk, Philadelphia, PA 19104; e-mail: weih@uphs.upenn.edu

Supported by Grant 12-FY05-62 from the March of Dimes Birth Defects Research Foundation, White Plains, NY (H.W.), K08 Grant (1-K08-GM073224-03) and R01 Grant (1-R01GM084979-01) National Institute of General Medical Science (NIGMS), National Institute of Health (NIH), Baltimore, Maryland (H.W.). Medical Student Anesthesia Research Fellowship (K.P.), Foundation of Anesthesia Education and Research (FAER), Rochester, MN.

The first two authors contributed equally to this project.
Because anesthetics easily cross the placenta, these interventions expose developing fetal brains to inhalational anesthetics as well. Because neurons in the developing brains are especially vulnerable to anesthesia-mediated neurodegeneration (9), it is possible that inhalational anesthetics administered to pregnant mothers can harm the developing fetal brains (14). We have previously shown that $1.3 \%$ isoflurane administered during late pregnancy did not produce detectable injury to the rat fetal brains (15). However, in the case of fetal surgery used to correct various congenital malformations during midgestation (18-25 wk) (16), the fetal brain can be exposed to 2-3 times (2.5-3 minimal alveolar concentration [MAC]) the normally used clinical concentrations of inhaled anesthetics to both relax uterine smooth muscle and provide adequate anesthesia (16). Fetal surgery is a rapidly growing and evolving area and may become the standard therapy for most disabling malformations that are usually treated in young infants $(16,17)$. Considering the dose-dependent characteristics of anesthesia-mediated neurodegeneration in both cell cultures $(2,18)$ and animal models $(9)$, it is important and urgent to know whether the high concentrations of inhalational anesthetics normally used during fetal surgery will cause neurodegeneration in the developing fetal brain. Following up on the use of a low concentration of isoflurane in our previous study (15), this study further investigated whether the high concentrations of isoflurane inhaled by pregnant mothers during fetal surgery would cause neurodegeneration in developing fetal rat brains.

\section{METHODS}

Animals. The Institutional Animal Care and Use Committee (IACUC) at the University of Pennsylvania approved all experimental procedures and protocols used in this study. All efforts were made to minimize the number of animals used and their suffering. Sprague-Dawley pregnant rats (Charles River Laboratories, Wilmington, MA) were housed in polypropylene cages, and the room temperature was maintained at $22^{\circ} \mathrm{C}$, with a 12 -h light-dark cycle. Pregnant rats at gestational d 21 (E21) were used for all experiments, as in our previous study (15), because this time corresponds approximately to mid-gestation in human beings $(9,19)$ and is a common time for most fetal surgeries (18-25 wk) (16).

Experimental protocol. Our previous study demonstrated that $1.3 \%$ isoflurane for $6 \mathrm{~h}$ is the maximum exposure that would not cause a detrimental effect on arterial blood gas values, without tracheal intubation. For the purpose of the experimental design, we first performed a pilot study, using eight pregnant rats, to determine the maximum exposure time that $\sim 2$ MAC of isoflurane $(3 \%)$ can be used without decreasing arterial blood pressure and

Abbreviations: ABG, arterial blood gas; MAC, minimal alveolar concentration; MAP, mean arterial blood pressure 


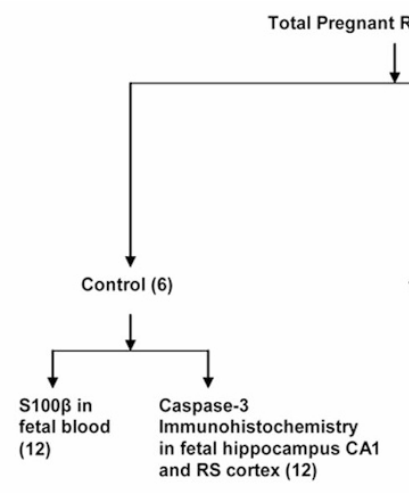

Rats E21 (18)

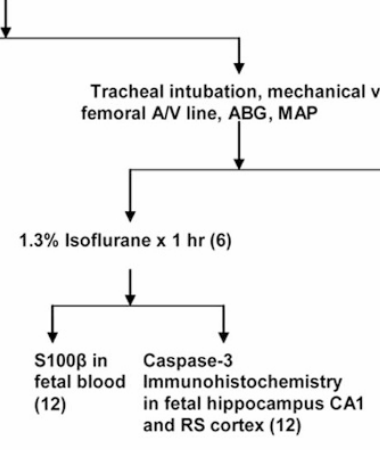

worsening arterial blood gas (ABG) values, even with tracheal intubation and mechanical ventilation. The results from our pilot study indicated that a maximum duration of exposure of $3 \%$ isoflurane was $1 \mathrm{~h}$, and that the $100 \%$ oxygen was also necessary to prevent hypoxia during the treatment. Therefore, in our formal experimental design as demonstrated in Figure 1, we divided the animals into three groups: control, low concentration of isoflurane $(1.3 \%)$, and high concentration of isoflurane (3\%) treatment groups. Pregnant rats in the control group inhaled only humidified $100 \% \mathrm{O}_{2}$ for $1 \mathrm{~h}$ in a chamber, with monitoring and maintenance of their rectal temperature at $37 \pm$ $0.5^{\circ} \mathrm{C}$, using a deltaphase isothermal pad (Braintree Scientific, Inc., Braintree, MA). The oxygen and carbon dioxide levels in the chamber were also monitored and maintained using a Datex-Ohmeda Capnomac Ultima ULT1A-27-08 anesthesia monitor (Instrumentarium Corp., Helsinki, Finland) as described in our previous study (15). Pregnant rats in the treatment groups were first anesthetized with inhalation of $1.3 \%$ isoflurane, and their trachea were intubated with an endotracheal tube (i.v. angiocatheter \#14), followed by mechanical ventilation, using an MR1-1 ventilator (CWE, Inc., Ardmore, PA). The rectal temperature was monitored continuously throughout the experiment by a thermometer (Fischer Scientific, Pittsburgh, PA) for both treatment groups and was maintained at $37 \pm 0.5^{\circ} \mathrm{C}$, using a Hospital Heating Pad (The Davol Rubber Company, Providence, RI). An arterial catheter was placed in the left femoral artery for continuous monitoring of mean arterial blood pressure (MAP) and collection of blood samples for the arterial blood gases (ABG). We obtained baseline MAPs and ABGs in pregnant rats after placement of an arterial catheter in the left femoral artery while the animals were anesthetized with $1.3 \%$ isoflurane. Thereafter, pregnant rats in the two treatment groups inhaled either low $(1.3 \%)$ or high $(3 \%)$ concentrations of isoflurane for $1 \mathrm{~h}$. The MAP was continuously monitored throughout the experiments and the arterial blood from the left femoral arterial catheter was collected periodically to determine ABG values. For pregnant rats receiving the $3 \%$ isoflurane treatment, occasional i.v. bolus injections of phenylephrine at $0.3 \mathrm{mg} / \mathrm{kg}$ via the left femoral vein were used to maintain MAP and ABG values within the normal physiologic range, without significant differences comparing to the low concentration (1.3\%) isoflurane treatment (Table 1; Fig. 2). These injections mimic a scenario of clinical practice during fetal surgery. Because, in obstetrics, phenylephrine is the recommended vasopressor to maintain arterial blood pressure within normal limits (20) and is an important factor in maintaining uterine blood flow. To our knowledge, no study has clearly demonstrated that phenylephrine itself can induce neurodegeneration in developing fetal brains. Blood glucose was simultaneously measured with a glucometer (ACCU-CHECK Advantage, Roche Diagnostics Corporations, Indianapolis, IN) to detect and treat hypoglycemia. Our previous study demonstrated that isoflurane administered to pregnant mothers quickly passes the blood-brain barrier and that isoflurane concentrations measured in the fetal
Figure 1. Nomogram illustrating distribution of pregnant mothers and their fetuses among different experimental groups.

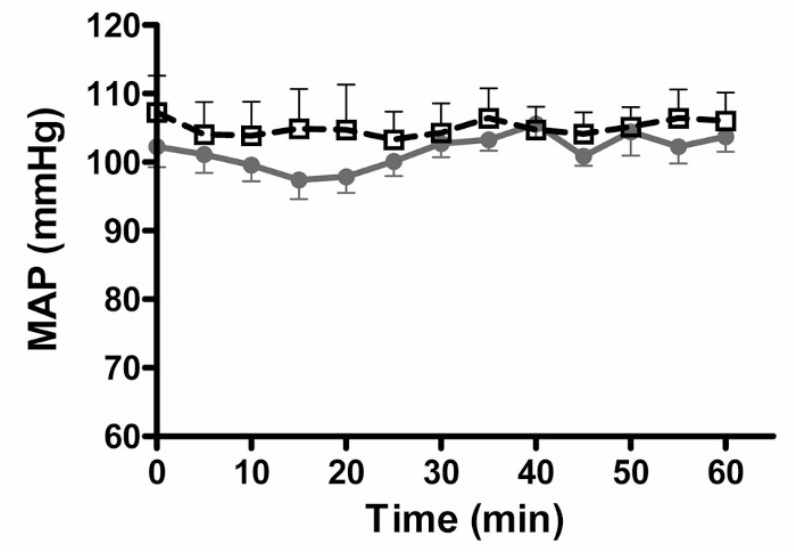

Figure 2. Changes of MAP during the treatment of isoflurane at different concentrations. Solid circle, $3 \%$ isoflurane; open square, $1.3 \%$ isoflurane. $n=$ 6 for each time point.

brains rapidly equilibrated with the inhaled isoflurane concentrations of their pregnant mothers (15). We did not further measure the concentrations of isoflurane in the fetal brains for this study.

Blood and brain tissue preparation. At $6 \mathrm{~h}$ after cessation of $1 \mathrm{~h}$ of either 1 or $3 \%$ isoflurane treatment or $100 \%$ oxygen inhalation in the case of control group, pregnant rats were anesthetized with sodium pentobarbital intraperitoneally (i.p. $100 \mathrm{mg} / \mathrm{kg}$ ), and the pups were delivered via cesarean section. Two pups from each pregnant mother were randomly chosen for obtaining blood and brain tissue to study the extent of neurodegeneration. We elected to perform the C-section for obtaining fetal blood and brains at $6 \mathrm{~h}$ after cessation of $1 \mathrm{~h}$ anesthesia treatment because the developing brains of newborn rodents clearly demonstrated anesthesia-induced early neurodegeneration about 5-8 h after onset of anesthesia treatment in previous studies. Therefore, $7 \mathrm{~h}(1 \mathrm{~h}$ anesthesia treatment plus $6 \mathrm{~h}$ postanesthesia duration) after beginning anesthesia treatment was considered a sensitive point in time to detect early neurodegeneration and was used in this study $(9,15)$. Pentobarbital has widely been used for the sacrifice of animals and should not significantly influence the extent of neurodegeneration in this study because the brains were removed immediately after the use of pentobarbital. Blood

Table 1. Arterial blood gas measurements

\begin{tabular}{|c|c|c|c|c|c|c|}
\hline & \multicolumn{2}{|c|}{ Baseline } & \multicolumn{2}{|c|}{$30 \mathrm{~min}$} & \multicolumn{2}{|c|}{$60 \mathrm{~min}$} \\
\hline & $1.3 \%$ ISO & $3.0 \%$ ISO & $1.3 \%$ ISO & $3.0 \%$ ISO & $1.3 \%$ ISO & $3.0 \%$ ISO \\
\hline $\mathrm{pH}$ & $7.45 \pm 0.02$ & $7.41 \pm 0.02$ & $7.44 \pm 0.04$ & $7.36 \pm 0.02$ & $7.37 \pm 0.03$ & $7.30 \pm 0.01$ \\
\hline $\mathrm{PaCO}_{2}(\mathrm{~mm} \mathrm{Hg})$ & $41.1 \pm 2.01$ & $40.9 \pm 1.75$ & $40.8 \pm 2.03$ & $45.3 \pm 4.97$ & $45.4 \pm 4.66$ & $45.3 \pm 3.01$ \\
\hline $\mathrm{PaO}_{2}(\mathrm{~mm} \mathrm{Hg})$ & $328.9 \pm 29.5$ & $364.1 \pm 28.4$ & $347.5 \pm 46.3$ & $340.5 \pm 37.0$ & $369.7 \pm 39.7$ & $362.2 \pm 42.6$ \\
\hline $\mathrm{HCO}_{3}{ }^{-}(\mathrm{mmol} / \mathrm{L})$ & $26.9 \pm 1.25$ & $26.6 \pm 1.17$ & $26.8 \pm 1.15$ & $26.7 \pm 2.80$ & $27.9 \pm 1.35$ & $26.9 \pm 1.27$ \\
\hline $\mathrm{BG}(\mathrm{mg} / \mathrm{dL})$ & $89.2 \pm 2.24$ & $98.6 \pm 3.54$ & $91.2 \pm 3.24$ & $103.8 \pm 7.26$ & $86.0 \pm 5.74$ & $96.0 \pm 6.06$ \\
\hline
\end{tabular}

Values are mean \pm SE. $N=6$ for each group.

ISO, isoflurane. 
$(0.1 \mathrm{~mL})$ was collected from the fetal left ventricle for determination of $\mathrm{S} 100 \beta$, a recommended useful biomarker for fetal brain damage during pregnancy (21). Thereafter, the fetal brains were perfused transcardially with ice-cold normal saline followed by 4\% paraformaldehyde in PBS (pH 7.4). The brains were then removed and postfixed overnight in the same fixative at $4^{\circ} \mathrm{C}$ and cryoprotected in $30 \%$ (wt/vol) sucrose at $4^{\circ} \mathrm{C}$ for $24 \mathrm{~h}$. The brains were then frozen in isopentane at $-20^{\circ} \mathrm{C}$ and stored at $-80^{\circ} \mathrm{C}$ until use. Serial coronal sections $(10 \mu \mathrm{m})$ were cut in a cryostat (Dolbey-Jamison Optical Company, Inc., Pottstown, PA), then mounted on gelatin-coated slides and stored at $-80^{\circ} \mathrm{C}$ until use.

Determination of $S 100 \beta$ in fetal blood. $\mathrm{S} 100 \beta$ levels in blood may be a useful predictor of fetal brain damage during pregnancy (21). We measured S100 $\beta$ levels in the fetal blood, using Sangtec 100 ELISA kits (DiaSorin, Inc., Stillwater, MN), following the manufacturer's protocol as described previously (22). Briefly, $50 \mu \mathrm{L}$ of plasma was placed in each well of a 96-well plate and mixed, with $150 \mu \mathrm{L}$ tracer from kit and incubated for $2 \mathrm{~h}$. This was then followed by $\left(3,3^{\prime}, 5,5^{\prime}\right.$ tetramethylbenzidine, TMB) substrate and stop solution. The OD was read at $450 \mathrm{~nm}$. The sensitivity was determined by plotting the standard curve and concentration, using it to determine the concentrations of the samples. We used only two fetal blood samples for each pregnant mother because of difficulties in obtaining enough blood $(0.1 \mathrm{~mL})$ from every fetus for adequate measurement of the $\mathrm{S} 100 \beta$ level.

Immunohistochemistry for caspase-3. Caspase-3-positive cells were measured in the hippocampal CA1 region and retrosplenial cortex, using immunohistochemical methods that we and others have described previously (15). These two brain regions were chosen because they are particularly vulnerable to anesthesia-mediated neurodegeneration $(9,15)$ and are important to memory and learning. Briefly, brain sections were first incubated in $3 \%$ hydrogen peroxide in methanol for 20 min to block endogenous peroxidase activity. Sections were then incubated with blocking solution containing $10 \%$ normal goat serum in PBS containing 0.1\% Tween 20 (PBST) for $1 \mathrm{~h}$ at room temperature after washing with $0.1 \%$ PBST. The antiactivated caspase-3 primary antibody (1/200, Cell Signaling Technology, Inc., Danvers, MA) was then applied in blocking solution and incubated at $4^{\circ} \mathrm{C}$ overnight. Tissue sections were biotinylated with goat anti-rabbit antibody (1/200, Santa Cruz Biotechnology, Inc., Santa Cruz, CA) in $0.1 \%$ PBST for 40 min, followed by incubation with the avidin-biotinylated peroxidase complex (Vectostain ABC-Kit, Vector Lab, Burlingame, CA) for $40 \mathrm{~min}$. Tissue sections were colorized with diaminobenzidine (DAB, Vector Laboratories, Burlingame, CA) for $8 \mathrm{~min}$ and counterstained with modified hematoxylin. Negative control sections were incubated in blocking solution that did not contain primary antibody. Images were acquired and assessed at $200 \times$ using IP lab 7.0 software linked to Olympus IX70 microscope (Olympus Corporation, Japan) equipped with a Cooke SensiCam camera (Cooke Corporation, Romulus, MI). Three brain tissue sections at $10 \mu \mathrm{m}$, corresponding to the Atlas of the Developing Rat Nervous System, Figure 105 (23), were chosen from each animal and analyzed for caspase-3-positive cells in the two brain regions. Two individuals blinded to the treatments counted the total number of caspase-3-positive cells in the hippocampal CA1 region and retrosplenial cortex. The areas of the hippocampus CA1 region and retrosplenial cortex were defined according to the Atlas of the Developing Rat Nervous System, Figure 105 (23) and the area measured using IPLab Suite v3.7 imaging processing and analysis software (Biovision Technologies, Exton, PA; www. BioVis.com.). The density of caspase-3-positive cells in a particular brain region was calculated by dividing the number of caspase-3-positive cells by the area of that brain region.

Statistical analysis. We averaged the data from two fetuses randomly chosen from the same mother and used this average as a single sample for all the data analysis derived from the fetuses to reduce variance from differentsized litters as was also done for our previous study (15). Six pregnant mothers were used for each group of anesthesia treatments, therefore the $n$ value used for the statistical analysis of MAP and ABG values for each experimental group was 6 . Because the fetal data were averaged from two fetuses from the same mother to form one data point for the blood $\mathrm{S} 100 \beta$ levels and caspase- 3 densities in the different brain regions, the $n$ value was also 6. Results of the ABG and MAP measurements in the pregnant rats were analyzed using two-way ANOVA repeat measurements because of different time points. Data for $\mathrm{S} 100 \beta$ levels and caspase-3 densities in the different fetal brain regions were analyzed using one-way ANOVA, followed by Tukey post hoc multiple comparison tests. In all experiments, differences were considered statistically significant at $p<0.05$.

\section{RESULTS}

Comparison of basic physiologic variables of $A B G$ and MAP. As shown in Table 1 and Figure 2, we maintained ABG values and MAP within the normal physiologic range in the treatment groups receiving either 1.3 or $3 \%$ isoflurane. This was done with i.v. injections of phenylepherine for the group receiving 3\% isoflurane and the use of $100 \%$ oxygen as gas carrier, mimicking the clinical scenario of fetal surgery. There were no significant differences between the low and high concentrations of isoflurane treatment groups on any measured variables for ABG and MAP ( $n=6$ for both). Taking these measures reduces the possibility that isoflurane-induced neurodegeneration in the fetal brains was caused by physiologic side effects (e.g. hypotension, hypoxia and hypercapnia).

The higher concentration of isoflurane increased $S 100 \beta$ levels in fetal blood. Although not statistically significant, the average blood level of $\mathrm{S} 100 \beta$ in the fetal rat whose mothers received $1.3 \%$ isoflurane treatment for $1 \mathrm{~h}$ was slightly higher than in the control group (Fig. 3). However, the average blood level of $\mathrm{S} 100 \beta$ in the fetus whose mothers were treated with $3 \%$ isoflurane for $1 \mathrm{~h}$ was significantly increased compared with the $\mathrm{S} 100 \beta$ level in the control group (121\% increase over the control level, $p<0.05, n=6$ ).

The higher concentration of isoflurane increased apoptosis in the developing fetal brains. The $1.3 \%$ isoflurane for $1 \mathrm{~h}$ did not significantly affect the density of caspase-3-positive cells in the CA1 region of the hippocampus (Fig. 4) or retrosplenial cortex (Fig. 5) when compared with the control. However, $3 \%$ isoflurane for $1 \mathrm{~h}$ significantly increased caspase- 3 densities in both the hippocampal CA1 region (Fig. 4, 115\% increase over the control, $p<0.05$ ) and in the retrosplenial cortex (Fig. 5, $154 \%$ increase over control, $p<0.001, n=6$ ).

\section{DISCUSSION}

Our study demonstrated that isoflurane exposure at a high concentration (3\%) in pregnant rats increased the blood levels of $\mathrm{S} 100 \beta$ in their fetuses, as well as increased the degree of apoptosis in the fetal hippocampal CA1 region and retrosplenial cortex of the fetal brains. These two brain regions are closely related to memory and learning and are particularly

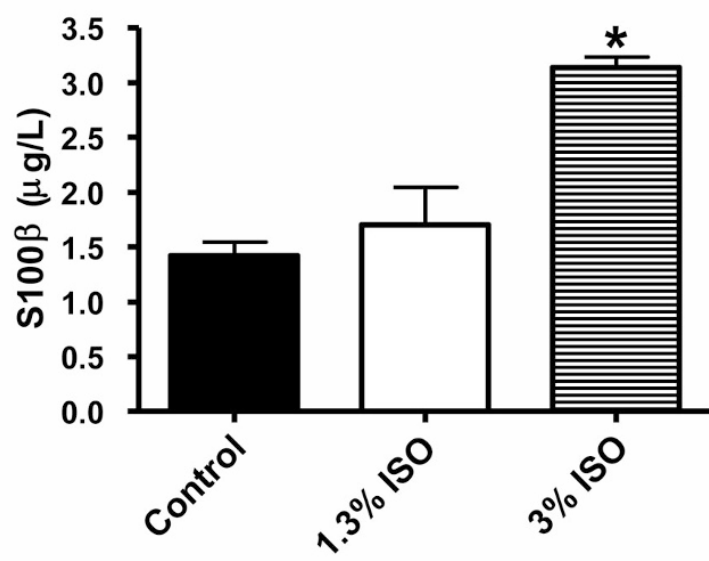

Figure 3. Isoflurane at high concentration (3\%) increased $\mathrm{S} 100 \beta$ in the rat fetal blood. $\mathrm{S} 100 \beta$ levels in the blood obtained from left ventricle puncture were determined using a Sangtic 100 ELISA kit. ISO, isoflurane. Data represent mean $\pm \mathrm{SE}$ from 12 rat fetus. ${ }^{*} p<0.05$ compared with both control and $1.3 \%$ ISO. 
A
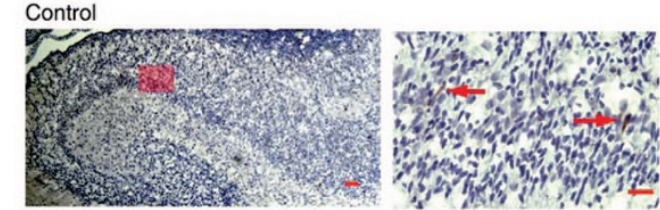

$1.3 \%$ Isoflurane $\times 1 \mathrm{hr}$
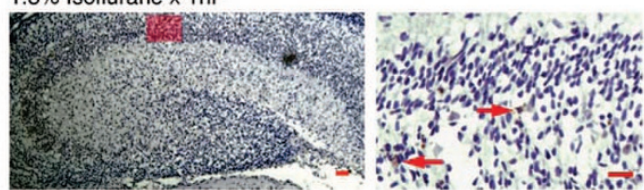

$3 \%$ Isoflurane $\times 1 \mathrm{hr}$

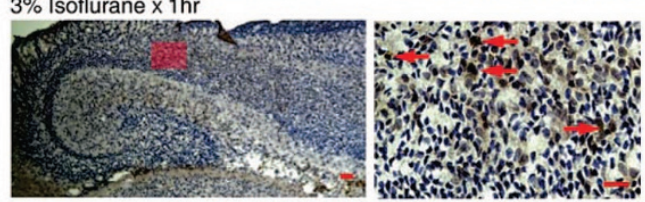

B

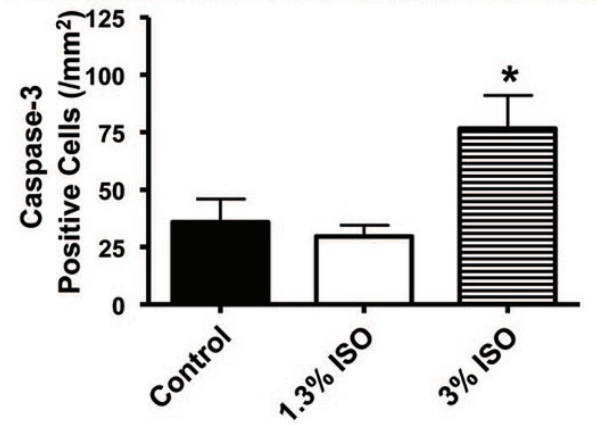

Figure 4. In utero isoflurane at high concentration (3\%) increased apoptosis in the hippocampus CA1 region of the fetal developing brains. (A) Arrows indicate caspase-3-positive cells in the hippocampus CA1 region located within the red rectangles illustrated on the left, demonstrating the entire hippocampus of developing fetal brains at low magnification. Scale bar $=50$ $\mu \mathrm{m}$ for the low magnification on the left and $10 \mu \mathrm{m}$ for the high magnification on the right (red bar on the right lower corner for each picture). (B) Data represent the mean $\pm \mathrm{SE}$ of 12 fetal rat brains from six pregnant mothers $(n=$ 6) in either the control group or the treatment groups with 1.3 or $3 \%$ isoflurane (ISO). ${ }^{*} p<0.05$ compared with both control and $1.3 \%$ ISO, $n=6$.

vulnerable to anesthesia-mediated neurodegeneration $(9,15)$. Similar to studies focusing on the fetal brains of guinea pigs (14) and the developing neonatal brains of rodents $(9,10)$, these results indicate that isoflurane at a higher concentration is capable of causing neurodegeneration in fetal rat brains.

The effects of anesthesia on apoptosis in the developing fetal brain have been controversial, with inhibition (15), no effects (24), and promoting effects (14) all being reported. Similarly, the effects of anesthesia used during the development of fetal or newborn brains on long-term memory and learning are also mixed with anesthesia being found to cause both transient improvement (15) and permanent impairment (9). Controversial data also demonstrated the improvement (25) and impairment (11) of cognitive function after adult rodents received general anesthesia. Together with our previous study (15), these results suggest that isoflurane induces neurodegeneration in the fetal rat brain only when it was used in higher concentrations ( $3 \%$ or around $2 \mathrm{MAC}$ ). These results are consistent with the dose-dependent toxic effects of isoflurane in tissue cultures $(2,7)$ and newborn rats (9). It should be noted that isoflurane induced a much greater degree of neurodegeneration in 7-d-old rats (9) than in the developing fetal
A

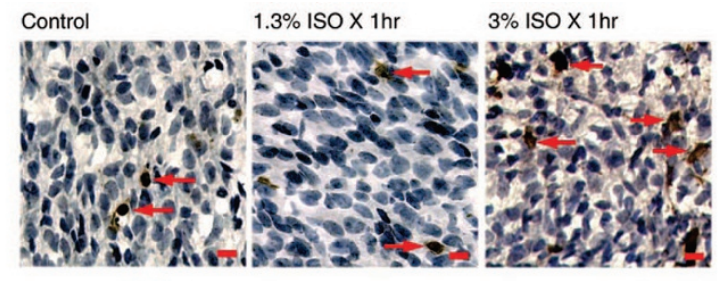

B

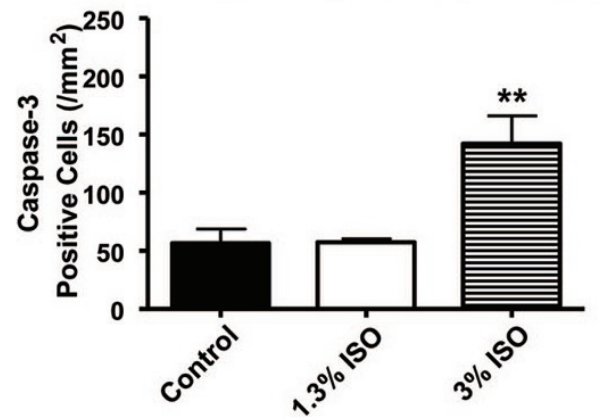

Figure 5. In utero isoflurane at high concentration (3\%) increased apoptosis in the retrosplenial cortex of the fetal developing brains. (A) Arrows indicate caspase-3-positive cells in the retrosplenial cortex in different experiment groups. (B) Data represent the mean $\pm \mathrm{SE}$ of 12 fetal rat brains from six pregnant mothers $(n=6)$ in either the control group or the treatment groups with 1.3 or $3 \%$ isoflurane (ISO). Scale bar $=10 \mu \mathrm{m}$ (red bar on the right lower corner for each picture). $* * p<0.001$ compared with both control and $1.3 \%$ ISO, $n=6$.

rat brains, demonstrated in the current study. It is hypothesized that synaptogenesis in rats peaks around postnatal $\mathrm{d} 7$, which may contribute to the increased vulnerability of the developing brain of 7-d-old rats to isoflurane-mediated neurodegeneration $(9,19)$. It has been proposed that neurons during periods of rapid growth or synaptogenesis in the developing brain are vulnerable to anesthesia-mediated neurodegeneration (9). The fact that a combination of isoflurane with nitrous oxide and midazolam was used in a previous study (9) may have also contributed to the increased neurodegeneration mediated by general anesthesia than that observed from isoflurane as a single agent in this study. Our recent study examining tissue cultures indicated that isoflurane can take on the properties of a "double-edged sword" in that it can be both neuroprotective and neurotoxic (18). Isoflurane at a low concentration and short duration seemed to precondition neurons and provide neuroprotection, whereas isoflurane at a high concentration for long duration directly induced neurotoxicity (18). Thus, $1.3 \%$ isoflurane inhaled by pregnant rats transiently inhibited spontaneous apoptosis in the fetal brain, possibly by preconditioning as seen in our previous study (15), whereas $3 \%$ isoflurane inhaled by pregnant mothers in this study induced apoptosis in the fetal rat brains. Given that spontaneous apoptosis in the developing fetal brain is a normal process that shapes the brain as it matures, the significance of increased apoptosis induced by anesthesia in developing brains is unclear, but, as demonstrated previously, it is likely to be detrimental to subsequent cognitive ability (9).

The developing brains of different animal species demonstrate varying vulnerabilities to anesthesia-mediated apoptosis. Isoflurane at a relatively low concentration $(0.55 \%)$ was 
shown to induce significant apoptosis in multiple brain regions in fetal guinea pigs (14), whereas a significantly higher concentration (3\%) of isoflurane was needed to induce apoptosis in the fetal rat brains in this study. The periods of synaptogenesis are sustained for a longer portion of the pregnancy in guinea pigs than in rats (19), potentially making the developing rat brains relatively less vulnerable to isoflurane toxicity compared with guinea pigs. However, isoflurane at the relatively high concentrations commonly used during fetal surgery could still induce significant apoptosis even in the fetal rat brains, which are theoretically less vulnerable to isoflurane-induced neurodegeneration. Together with the reports from other animal studies $(9,10)$, this investigation suggests that the administration of high concentrations of inhalational anesthetics to pregnant mothers may not be prudent, if these results in rodents can be translated to humans $(9,10)$.

An interesting finding in this study is that $\mathrm{S} 100 \beta$ levels in fetal blood significantly increased after pregnant mothers inhaled $3 \%$ but not $1.3 \%$ isoflurane. Unlike the diagnosis of myocardial infarction using troponin levels in the blood, the diagnosis of neurodegeneration in the CNS is usually difficult because no such selective and sensitive biomarkers for neurodegeneration have been well developed. S100 $\beta$ seems to be most abundant in glial cells in the nervous system and has been widely used as one of the biomarkers for neurodegeneration in CNS. S100 $\beta$ may be a useful biomarker for fetal brain damage during pregnancy because its concentration has been demonstrated to be increased in amniotic fluid and cord blood in those fetuses that experienced a brain insult during pregnancy (21). The results from this study support the hypothesis that $\mathrm{S} 100 \beta$ levels in fetal blood may be a useful biomarker for fetal brain damage, although its usefulness in detecting neurodegeneration in anesthesia-mediated brain damage in newborn and adult brains needs further investigation.

The mechanisms for inhaled anesthetic-mediated neurodegeneration in the developing brain are still not clear. Some studies suggest that activation of $\mathrm{GABA}_{\mathrm{A}}$ receptors and inhibition of NMDA receptors by anesthetics, such as isoflurane, may play an important role in the induction of neurotoxicity in the developing brain (9). Our recent studies, both in tissue culture and in animals, suggest that inhalational anesthetics induce apoptosis through a disruption of intracellular calcium homeostasis, particularly through the overactivation of $\mathrm{IP}_{3}$ receptors and abnormal calcium release from the endoplasmic reticulum $(2,3,8)$. In addition, it seems that cells with elevated $\mathrm{IP}_{3}$ receptor activity, such as rat pheochromocytoma neurosecretory (PC12) cells transfected with Alzheimer's presenilin-1 (L286V) mutation or Q-111 rat striatal neurons (a cell model of Huntington disease), were more vulnerable to isoflurane-mediated apoptosis and calcium release from the endoplasmic reticulum $(2,8)$. A better understanding of the mechanisms of anesthesia toxicity will help us to develop strategies that will minimize the possible harmful effects of general anesthesia to patients.

This study has several limitations. First, we did not assess the effects of a high concentration of isoflurane used during pregnancy on the postnatal cognitive function. Additionally, we did not perform any type of fetal surgery in this study, which may have effects on inflammation in the brains and affect postoperative cognitive function (26). We found it difficult to maintain $\mathrm{PaO}_{2}$ unchanged for more than $1 \mathrm{~h}$, even at $\mathrm{FiO}_{2}$ of $100 \%$ and mechanical ventilation, under the conditions of both deep anesthesia and i.v. phenylephrine infusion. Although injections of phenylephrine are often used in clinical patients to maintain MAP within the physiologic range during fetal surgery and no studies to date suggest that phenylephrine could affect neurodegeneration significantly, this remains a limitation. We also did not examine the effects of isoflurane exposure on apoptosis in the developing brains at other time points during the gestation period. In addition, the $n$ value for each group is relatively low but did demonstrate statistical significance because of the remarkable increase of $\mathrm{S} 100 \beta$ levels in the fetal blood and significantly increased apoptosis in the fetal hippocampus and retrosplenial cortex after treatment with a high concentration of isoflurane in pregnant mothers.

In conclusion, prenatal exposure of isoflurane at high (3\%), but not low (1.3\%), concentration induced neurodegeneration in the fetal rat developing brains.

Acknowledgments. We gratefully recognize the discussions with Roderic Eckenhoff, M.D., Professor of Anesthesia, Maryellen Eckenhoff, Ph.D., Research Associate, and Randall Pittman, Ph.D., Professor of Pharmacology, from the University Of Pennsylvania School of Medicine, Philadelphia, PA. We thank Dr. Christopher Ward from the Children Hospital of Philadelphia (CHOP) for editing the manuscript.

\section{REFERENCES}

1. Wise-Faberowski L, Zhang H, Ing R, Pearlstein RD, Warner DS 2005 Isofluraneinduced neuronal degeneration: an evaluation in organotypic hippocampal slice cultures. Anesth Analg 101:651-657

2. Wei H, Liang G, Yang H, Wang Q, Hawkins B, Madesh M, Wang S, Eckenhoff R 2008 The common inhalational anesthetic isoflurane induces apoptosis via activation of inositol 1,4,5-trisphosphate receptors. Anesthesiology 108:251-260

3. Yang H, Liang G, Hawkins BJ, Madesh M, Pierwola A, Wei HF 2008 Inhalational anesthetics induce cell damage by disruption of intracellular calcium homeostasis with different potencies. Anesthesiology 109:243-250

4. Xie Z, Dong Y, Maeda U, Moir RD, Xia W, Culley DJ, Crosby G, Tanzi RE 2007 The inhalation anesthetic isoflurane induces a vicious cycle of apoptosis and amyloid beta-protein accumulation. J Neurosci 27:1247-1254

5. Malledant Y, Siproudhis L, Tanguy M, Clerc C, Chesne C, Saint-Marc C, Guillouzo A 1990 Effects of halothane on human and rat hepatocyte cultures. Anesthesiology 72:526-534

6. Chang YC, Chou MY 2001 Cytotoxicity of halothane on human gingival fibroblast cultures in vitro. J Endod 27:82-84

7. Wei H, Kang B, Wei W, Liang G, Meng QC, Li Y, Eckenhoff RG 2005 Isoflurane and sevoflurane affect cell survival and BCL-2/BAX ratio differently. Brain Res 1037:139-147

8. Liang G, Wang QJ, Li Y, Kang B, Eckenhoff MF, Eckenhoff RG, Wei HF 2008 A presenilin-1 mutation renders neurons vulnerable to isoflurane toxicity. Anesth Analg 106:492-500

9. Jevtovic-Todorovic V, Hartman RE, Izumi Y, Benshoff ND, Dikranian K, Zorumski CF, Olney JW, Wozniak DF 2003 Early exposure to common anesthetic agents causes widespread neurodegeneration in the developing rat brain and persistent learning deficits. J Neurosci 23:876-882

10. Ma D, Williamson P, Januszewski A, Nogaro MC, Hossain M, Ong LP, Shu Y, Franks NP, Maze M 2007 Xenon mitigates isoflurane-induced neuronal apoptosis in the developing rodent brain. Anesthesiology 106:746-753

11. Culley DJ, Baxter MG, Yukhananov R, Crosby G 2004 Long-term impairment of acquisition of a spatial memory task following isoflurane-nitrous oxide anesthesia in rats. Anesthesiology 100:309-314

12. Wilder RT, Flick RP, Sprung J, Katusic SK, Barbaresi WJ, Mickelson C, Gleich SJ, Schroeder DR, Weaver AL, Warner DO 2009 Early exposure to anesthesia and learning disabilities in a population-based birth cohort. Anesthesiology 110:796804 
13. Kalkman CJ, Peelen L, Moons KG, Veenhuizen M, Bruens M, Sinnema G, de Jong TP 2009 Behavior and development in children and age at the time of first anesthetic exposure. Anesthesiology 110:805-812

14. Rizzi S, Carter LB, Ori C, Jevtovic-Todorovic V 2008 Clinical anesthesia causes permanent damage to the fetal guinea pig brain. Brain Pathol 18:198-210

15. Li Y, Liang G, Wang S, Meng Q, Wang Q, Wei H 2007 Effect of fetal exposure to isoflurane on postnatal memory and learning in rats. Neuropharmacology 53:942950

16. Myers LB, Cohen D, Galinkin J, Gaiser R, Kurth CD 2002 Anaesthesia for fetal surgery. Paediatr Anaesth 12:569-578

17. Goldsmith MF, Straus SE, Kupfer C, Lenfant C, Collins F, Hodes RJ, Gordis E, Fauci AS, Alexander D, Battey JF, Slavkin HC, Leshner AI, Olden K, Hyman SE, Fischbach GD 19992020 vision: NIH heads foresee the future. JAMA 282:22872290

18. Wei H, Liang G, Yang H 2007 Isoflurane preconditioning inhibited isofluraneinduced neurotoxicity. Neurosci Lett 425:59-62

19. Dobbing J, Sands J 1979 Comparative aspects of the brain growth spurt. Early Hum Dev 3:79-83
20. Ngan Kee WD, Khaw KS 2006 Vasopressors in obstetrics: what should we be using? Curr Opin Anaesthesiol 19:238-243

21. Michetti F, Gazzolo D 2003 S100B testing in pregnancy. Clin Chim Acta 335:1-7

22. Kofke WA, Konitzer P, Meng QC, Guo J, Cheung A 2004 The effect of apolipoprotein $\mathrm{E}$ genotype on neuron specific enolase and S-100beta levels after cardiac surgery. Anesth Analg 99:1323-1325

23. Paxinos G, Ashwell KW, Tork I 1995 Atlas of the Developing Rat Nervous System. 2nd Academic Press, San Diego

24. McClaine RJ, Uemura K, de la Fuente SG, Manson RJ, Booth JV, White WD, Campbell KA, McClaine DJ, Benni PB, Eubanks WS, Reynolds JD 2005 General anesthesia improves fetal cerebral oxygenation without evidence of subsequent neuronal injury. J Cereb Blood Flow Metab 25:1060-1069

25. Rammes G, Starker LK, Haseneder R, Berkmann J, Plack A, Zieglgansberger W, Ohl F, Kochs EF, Blobner M 2009 Isoflurane anaesthesia reversibly improves cognitive function and long-term potentiation (LTP) via an up-regulation in NMDA receptor 2B subunit expression. Neuropharmacology 56:626-636

26. Wan Y, Xu J, Ma D, Zeng Y, Cibelli M, Maze M 2007 Postoperative impairment of cognitive function in rats: a possible role for cytokine-mediated inflammation in the hippocampus. Anesthesiology 106:436-443 